\title{
Uterine Corpus Sarcoma by AJCC v8 Stage
}

National Cancer Institute

\section{Source}

National Cancer Institute. Uterine Corpus Sarcoma by A/CC v8 Stage. NCI Thesaurus.

Code C139869.

A term that refers to the staging of uterine corpus sarcoma according to the American Joint Committee on Cancer, 8th edition. This staging system applies to leiomyosarcomas, endometrial stromal sarcomas, and adenosarcomas of the uterine corpus. It does not apply to carcinosarcomas which are staged according to the carcinomas and carcinosarcomas of the uterine corpus. (from AJCC 8th Ed.) 